\title{
The Research of Visual Management System Based on 2.5 Dimensional Museum
}

\author{
Na Wang ${ }^{1}$, Yunna Zhu ${ }^{2}$ and Guiyang Liu $^{3}$ \\ ${ }^{1,3}$ College of Information Technology, Heilongjiang Bayi Agricultural University, \\ Daqing, Heilongjiang, 163319, China \\ ${ }^{2}$ College of Information and Communication Engineering, Liaoning equipment \\ manufacturing Vocational and Technical College, Shenyang, 110161, China \\ 1yaya588588@163.com, ${ }^{2}$ Zhuyn0407@163.com, ${ }^{3}$ guiyangliu@126.com
}

\begin{abstract}
At present, the digitalization of museums has two forms which are two-dimensional images and three-dimensional virtual. But the two-dimensional digital museum is not strong in threedimensional space and poor human-computer interaction; three dimensional virtual museums occupy a large space which is not conducive to the transmission of the shortcomings. In order to improve these problems, the design of the museum visual management system based on $2.5 \mathrm{D}$ is presented. This paper introduces the system design idea, the technical route, the acquisition method of cultural material, the establishment of various models, the dynamic loading of the scene map, the virtual character tracking algorithm and other key technologies.
\end{abstract}

Keywords: 2.5-Dimension, Museum management, Visualization

\section{Introduction}

With the development of science and technology in our country, people's demand for culture is getting higher and higher. Museum as a carrier to spread, preserve and display the history, culture, science, technology and social features, it has been paid attention to more and more people. A prominent issue is that how to reflect the museum display, education and research functions better, offer better service to the community and the public and promote the development of the museum. Today with the network information technology and the rapid development of science and technology, traditional placement, labels, instructions, pictures and other methods have been unable to meet people's requirements; the transmission of culture has a lot of limitations. The visual management of the museum which realized by the network technology will break the limitation of the existing Museum, visitors no longer adhere to the unilateral view but many aspects of the senses through human-computer interaction and virtual reality, and have a deeper understand of the meaning of traditional culture Chinese [1]. The visualization system of the museum breakthrough time and space constraints are between the museum and the visitors, so that visitors can get more autonomy, convenience, sense of immersion. The museum's collections, exhibitions and education functions can be extended thus shorten the distance between visitors and exhibitions [2]. When people acquire knowledge, the exhibitions can receive the greatest degree of protection at the same time. The visualization

Article history:

Received (December 13, 2019), Review Result (January 18, 2020), Accepted (February 26, 2020) 
system of the museum contributes to cultural retention and inheritance and plays the function of the museum in the maximum extent.

At present, the two-dimensional visual management system of the museum has become to mature. It has the characteristics of small data amount and fast transmission speed and so on. But the display part is the form of two-dimensional picture of the exhibits in the museum, stereo sense is less strong support, and poor human-computer interaction, it cannot arouse visitors 'interests. Now three-dimensional digital museum has developed, such as the Shanghai World Expo Qingming Rivera true representation of the history of the Northern Song Dynasty Kaifeng that beyond time and space for the digital display of the Forbidden City, which is the first Chinese cultural heritage built virtual palace. Three dimensional digital museums give an immersive feeling [3], but it takes up large space, which is not conducive to the transmission of the network. So this paper attempts to study the construction of 2.5 dimensional visualization management systems with characteristics of two-dimensional and three-dimensional digital museum. Visualization management system based on the 2.5 dimensional museum is the combination of 2D and 3D scene to achieve the roaming scene design and integrated manage the scene of the relevant information.2.5-dimensional is a three-dimensional according to a certain projection rules mapped to a plane to show three-dimensional effect of the twodimensional graphics.2.5-dimensional museum visualization management system not only has the characteristics of two-dimensional space, easy to transfer, but also has a three-dimensional natural, real, rich spatial entity performance capabilities, which can be a good run on the network [4][5].

\section{System design}

With the development of network and virtual reality technology, more and more digital museums have been built, but there are still has some problems and shortcomings. Therefore, the paper considers the design of two-dimensional and three-dimensional advantages of the museum visual management system.

\subsection{System construction goals}

In order to develop a museum visualization management system which is suitable for network transmission and has a three-dimensional effect, the following objectives are determined:

(1) In the protection of the exhibits are not damaged circumstances, the establishment of the exhibits model to restore as high as possible;

(2) Navigation clear, convenient retrieval, easy to upgrade and easy to maintain;

(3) With the function of viewing the local details of the exhibits;

(4) When the virtual character walks in the scene, it has the function of collision detection and depth detection;

(5) Integrated application of multimedia display technology, 2D, 3D and network transmission technology.

\subsection{Design ideas}

In this paper, visualization system is considered to improve the current network on digital visual management deficiency of museum, and puts forward a new design idea, the system can realize the following functions: 
(1) Model management. Administrator for booth in the model to carry on the corresponding change, and can be illustrated relevant information to display (time, relics were unearthed in the level of cultural relics, description), such as changes in order to meet new museum exhibits functions.

(2) View the exhibits in all directions. When users come to a certain area whose the first views the small, one-dimensional view of the exhibit model. In order to allow the user to close watch displays, click on the up, down, left, right and expansion, narrow button, can undertake the corresponding operations.

(3) Any roaming. Users can walk in the exhibition hall arbitrary, when the route which the user clicks contains obstacles, the users can automatically bypass the obstacles to reach the destination.

(4) Realize switching of a plurality of virtual scenes. For the convenience of the user from one exhibition to another it, a pavilion to reach the entry and exit in the pavilion should be installed automatically triggers. So that it can realize multiple changes of virtual scene.

(5) Automatically trigger interpretation of cultural relics. In view of the need to explain the exhibits can trigger automatically triggers, when a user to cultural relics, can automatically trigger the explanation of cultural relics. It is similar to the effect of the infrared induction in real scene.

(6) With collision detection and depth detection function. When the virtual character in the scene movement encountered exhibits or other obstacles, the characters will not pass through, and according to its location to achieve occlusion effect.

(7) Rapid navigation. On the left side of the form, there is a tree structure, which is used to show that the exhibition area. When the user clicks the user can quickly reach the designated area on the right side.

(8) Chat forum. Users can communicate in here and leave the message, the administrator or some the full-time staff could answer the relevant questions.

(9) Release management information. The administrator can release some of the latest information about the museum through this function, so that users could understand the latest news about the museum.

\subsection{Adopt technical route}

The system mainly realizes the visual management of the museum, including digital museum and its information management. The museum's digital is included scenes of the digital and digital museum collections. The paper is to achieve the 2.5-dimensional digital museum, first realize the museum's three-dimensional virtual, and then mapped to 2.5-dimensional. Threedimensional virtualization of museum scenes created using Sketchup and 3ds Max software. Because most of the museum collections are very valuable, and cannot measurement directly many times, but also want to pursue the effect of high degree of reduction, so the text use reverse engineering modeling. First of all, through the three-dimensional scanner to obtain the geometry of the real object data, we cannot get the real model data after he completion of the first step of three-dimensional scan work. It needs the second step, post-processing mapping. Mapping process is the first target object of its type, get close to the photo. And then in the software, in the form of artificial, will receive the pictures paste to the model surface, to obtain a complete, colored geometric data. The three-dimensional model renders in 45 degree parallel projection, and then made of 2.5 dimensional model results. After the model has been synthesized, synthesis of large 2.5 dimensional grounds and exhibition scene, to achieve 
roaming, navigation and data management functions. The technical route of system design is shown in [Figure 1].

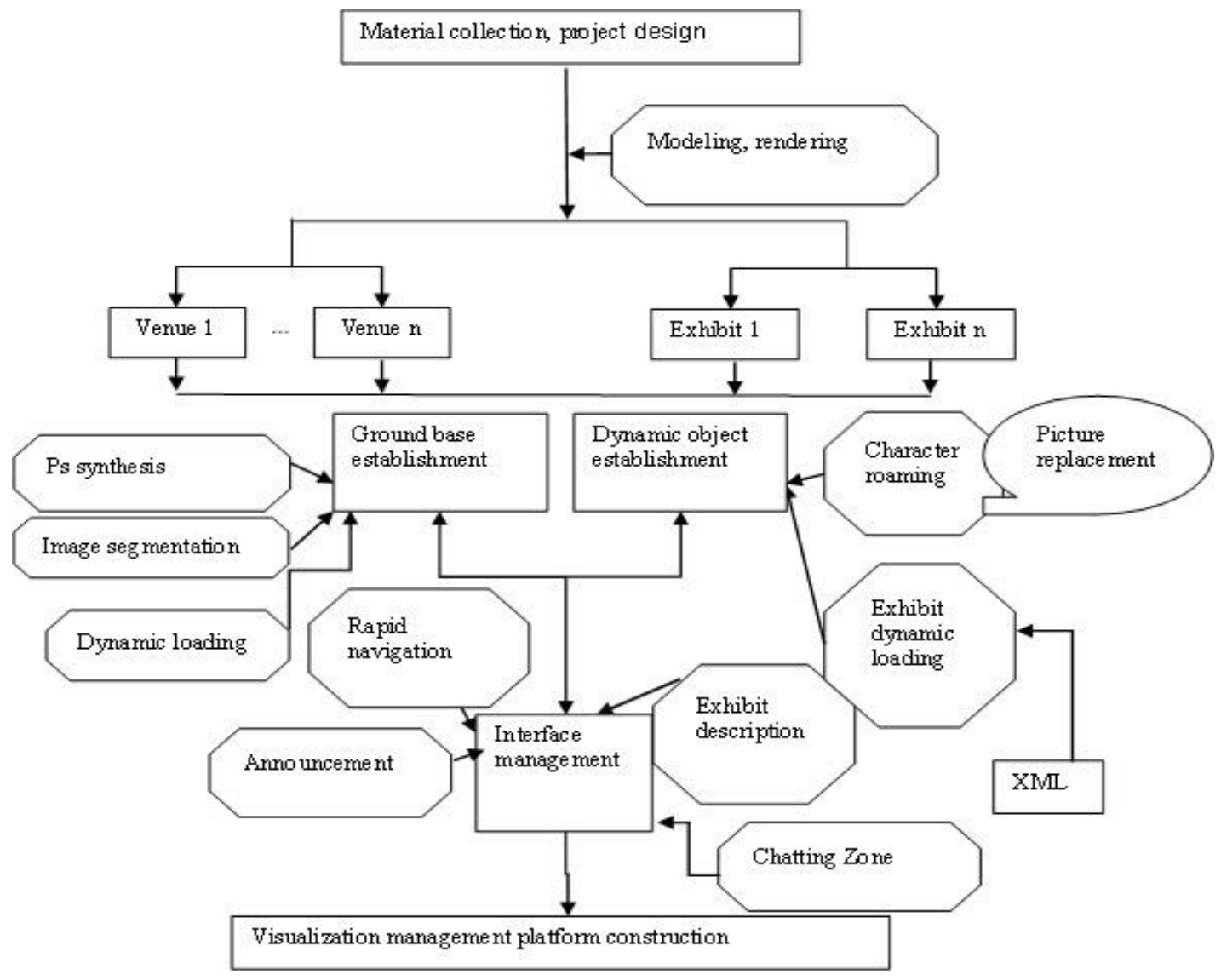

Figure 1. Technical route of system design

\section{The principle of key technologies}

\subsection{Acquisition of material of cultural relics}

The cultural relics are very precious. For the digital display of cultural relics, the reverse engineering technology is adopted. First, the texture, shape, color, surface texture, ornamentation and size of the cultural relics are collected by non-contact three-dimensional scanning measurement technology and then establish the model by the collected data. In order to achieve realistic results, rotating the object to shoot a large number of photos, How to shoot a large number of target object photos fast, intelligent is one aspect of the study. In this paper, design the automatic shooting device to get the photo. The automatic shooting device is shown in [Figure 2]. 


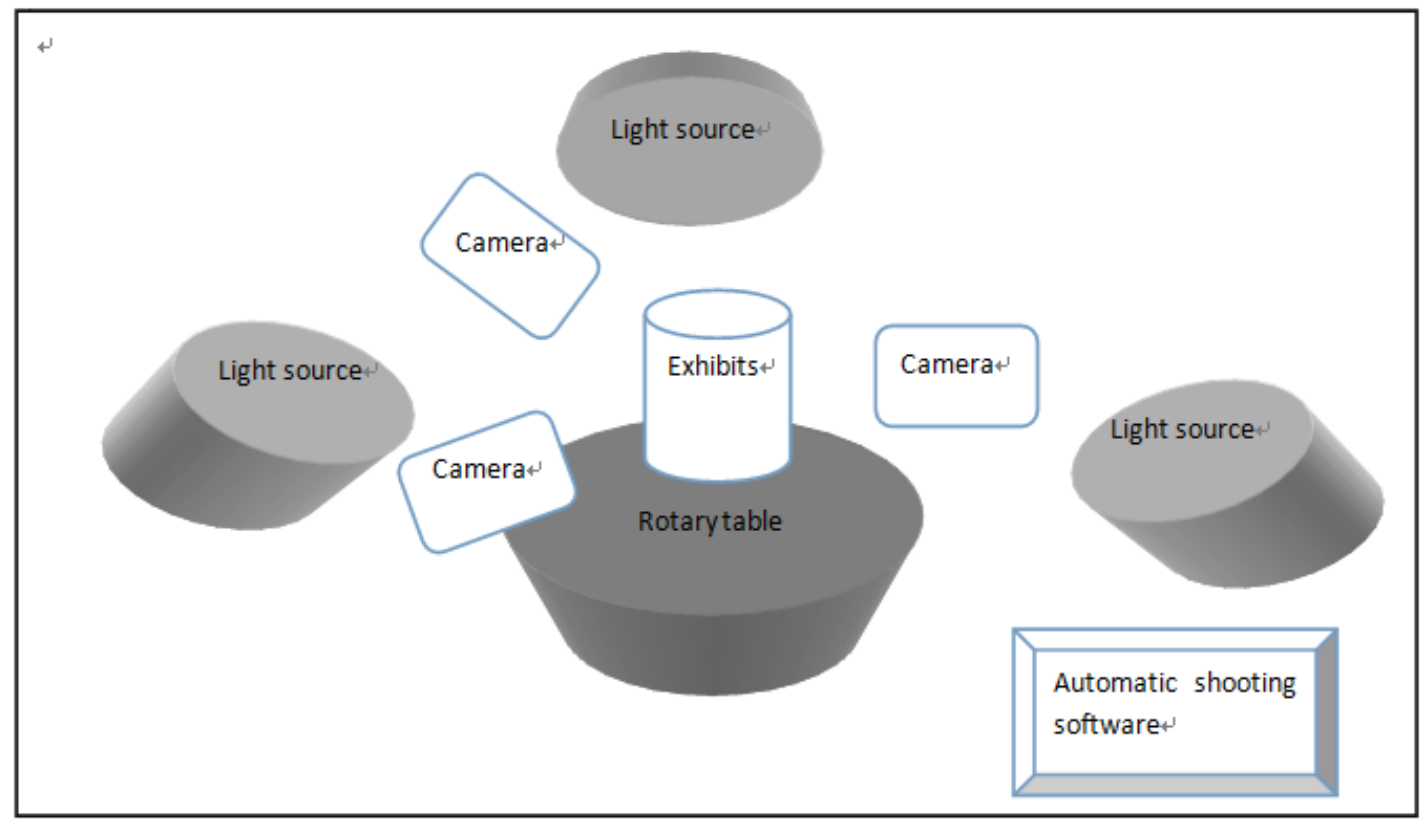

Figure 2. Automatic shooting device

The hardware of the automatic photographing device comprises a rotating device, a camera, and a light source. Rotating device consists of a rotating table and the composition of the relics; cultural relics fixed on the rotating table, adjust the focal length of the three cameras according to the size of the relics. Through the automatic shooting software control stepper motor operation, the rotation of the stepper motor driven to achieve the rotation of the main body and cultural relics, at the same time. The rotation of the turntable is controlled by a stepping motor of 0.025 , which automatically controls the motor to stop for 1 second each time the motor rotates 225 units and the three cameras to start shooting at the same time. Stop for 1 second each time is to make the camera more stable shooting, in order to ensure the quality of the shooting. The captured pictures are placed in three corresponding folders. The folder is named by the top 10 English letters of the relic (if inadequate, Tim a supplement) $+\mathrm{a}|\mathrm{b}| \mathrm{c}$ (A, b, c represent up middle down), The name of the file is 2-bit, sorted by the order in which it was taken. Each camera took 64 shots. The resulting image is processed using image processing software and then mapped. The use of automatic shooting system can quickly capture the required artifacts material picture, complete multiple angles of the shooting, which can save a lot of manpower [6][7].

\subsection{Building model production methods}

Establish the normal 3D model. The center of the bottom of the model is the origin of world coordinates $(0,0,0)$. As the system is 2.5 -dimensional simulation, so only make model in the top, front, left side. Place the camera in the direction of the object left 45 degrees, up 30 degrees direction, rendering renderings and ground perspective effect is basically the same. Take the camera and ground center distance of 200 meters as an example, create the target camera, camera coordinates $(\mathrm{x}, \mathrm{y}, \mathrm{z})$, camera target coordinates $(0,0,0)$. The calculating formula as follows:

$$
x=200 \times \cos 30^{\circ} \times \sin 45^{\circ}
$$




$$
\begin{gathered}
y=-x \\
z=200 \times \sin 30^{\circ}
\end{gathered}
$$

\subsection{5-dimensional scene map production methods}

Before the production need to establish a complete CAD drawings for the museum scene, build walls, the pavement edge at the same time, and export DXF format, import into CAD. Set the scene size consistent with the CAD drawings, a pixel corresponding to $1 \mathrm{~m}$, the DXF picture and scene alignment. Each area in the map make by Sketch Up. Sketch Up is known as the sketch master, simple, easy to use but post-model trimming, optimization, rendering need into the 3dMax software to deal with. And then use the principle of parallel projection, and finally use Photoshop to adjust the image and the final synthesis [8].

\subsection{Scene map dynamic loading}

Map relative to other museum scene is larger, if a static loading the entire scene, the time needed for longer, at the same time may appear the phenomenon of canton, thus affect your mood. This article adopts the method of dynamic loading, the image segmentation; the big scene map is divided into a number of square blocks, and through the index table map between the links [9][10] .

\subsubsection{Segment the large scene map into terrain blocks}

In this paper, how to cut large image rows and columns, and each small plot of width and height, and how to put a big scene images according to certain rules cut into several small images. The geometric relationship is shown in [Figure 3].

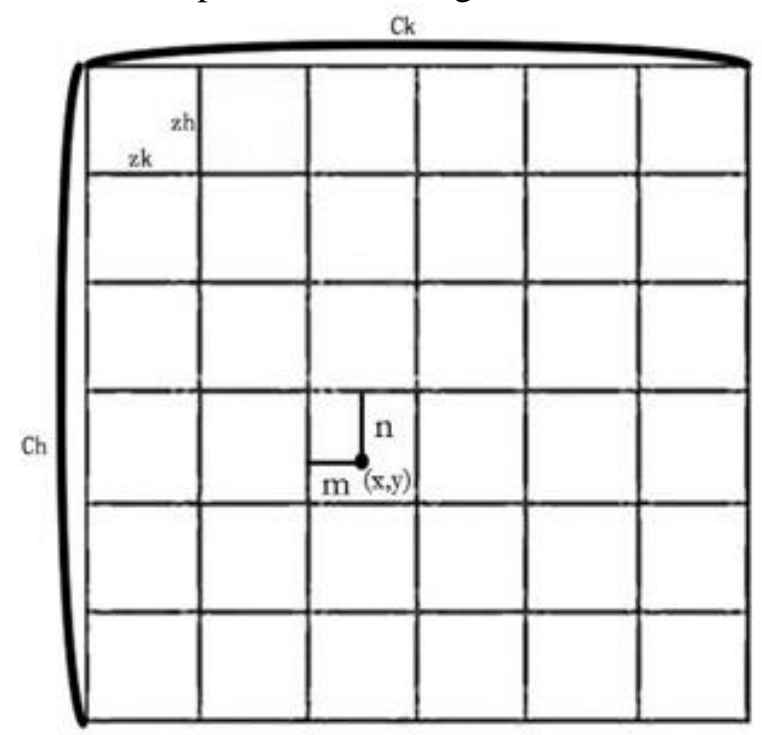

Figure 3. Geometric diagram

The main parameters of the mathematical model of image segmentation algorithm:

$R$ - How many rows of the image should be split;

$C h$-The height of the big image;

$z h$-The height of the small image; 


$$
R=C h / z h
$$

$C$ - How many columns the image should be split;

$C k$ - The width of the large image of the visual scene;

$z k$-The width of the small image;

$$
C=C k / z k
$$

$r l$-This row is the first few rows;

$x$-Point of abscissa

$m$ - How many columns are the front row in the small image;

$$
X=r 1 \times z h+m
$$

c2-Current column is the first of several columns;

$y$-Point of the vertical coordinates

$n-$ Current row is the first of several rows;

$$
y=c 2 \times z k+n
$$

Image segmentation algorithm theory: A large image is cut into $\mathrm{R}$ rows and $\mathrm{C}$ columns, to determine whether the current number of rows and columns beyond $\mathrm{R}$ or $\mathrm{C}$, if exceeded, exit the loop, if not, get the current location of the point. Whether the position of the current point is smaller than the width and height of the big image, if not, to get a small image from the relative position of the large image, otherwise out of the loop body. The cutting algorithm is shown in [Figure 4].

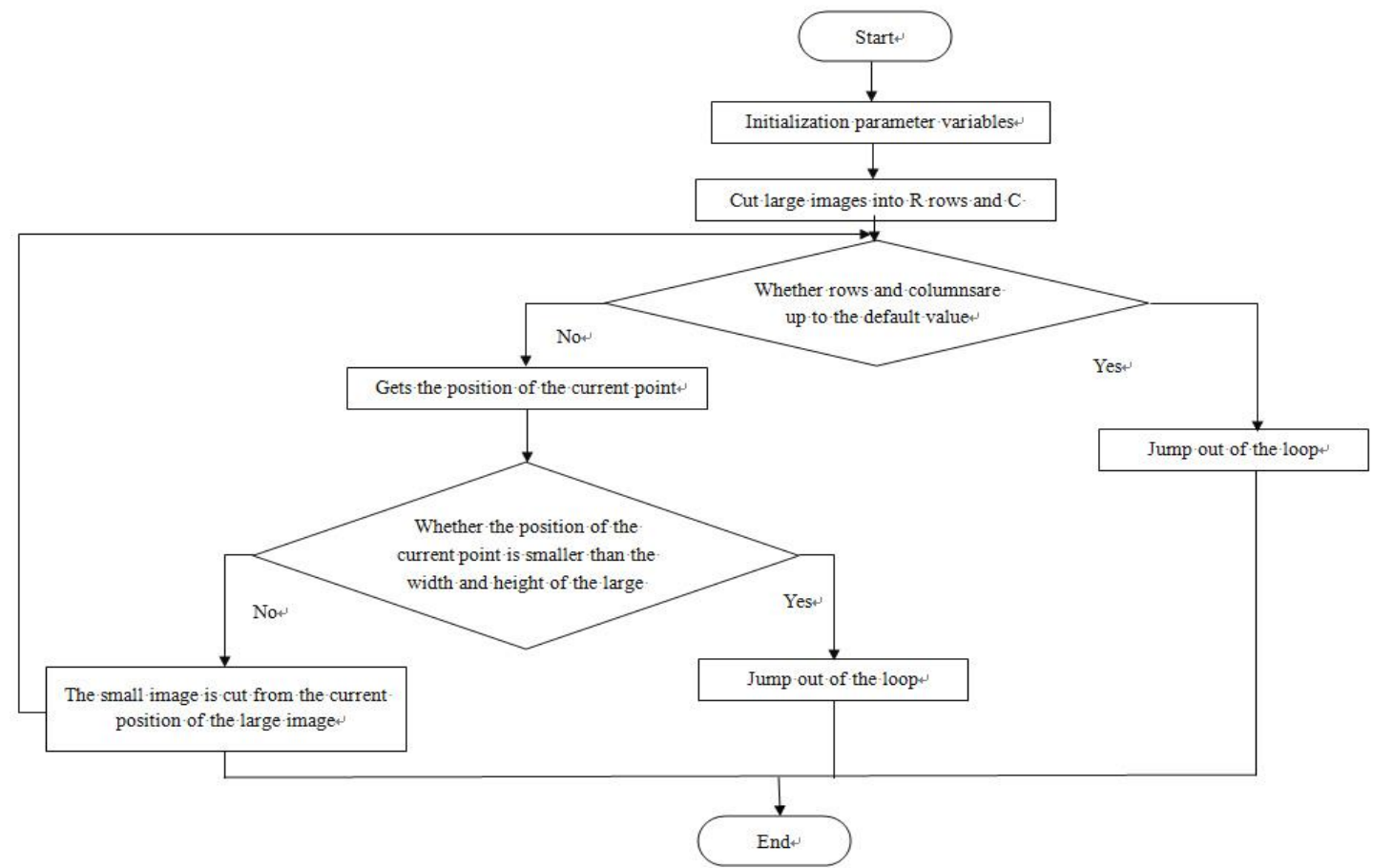

Figure 4. Cutting algorithm

In order to better display the contents of the museum scene, which set the size of each map area is 3584px*1792px. Each map area is composed of 98 small plots, which are arranged in the way of $14 * 7$. Because the setting of the browser window is $768 \mathrm{px} * 768 \mathrm{px}$, the display 
window cannot display the entire map area at a time. At the same time, in order to enable the viewer to browse the various locations of the museum, not card screen phenomenon occurs; the program needs to continue to preload the upcoming map area. In this paper, each map plot of a map area and the different map area are connected in series through the linked list [11][12]. In this paper, Using XML files to record the map area index, its location, and its surrounding eight plots index. As shown in [Figure 5], for the fifth plot, the plots index of NO.1, 2, 3, 4, 6, 7, 8, 9 need to be remember.

\begin{tabular}{|l|r|r|l|}
\hline 1 & $21^{\prime}$ & $32^{\prime}$ & $3^{\prime}$ \\
\hline 4 & $54^{\prime}$ & $65^{\prime}$ & 6 \\
\hline 7 & $87^{\prime}$ & $98^{\prime}$ & $9^{\prime}$ \\
\hline
\end{tabular}

Figure 5. Loading process of map area

\subsubsection{Dynamic loading of plots}

In order to clearly display the exhibits in the museum more clearly, the size of each venue map area is designed to be greater than the size of the form can be displayed. At the same time, there are many venues, there are many map areas. The load is divided into the same map region and the loading of different map regions.

When the virtual characters browse the same map area, the minimum distance between the character and the window boundary is set at first. When the virtual characters in the form of walking, walking distance is less than the minimum distance from the border, then moving the window, adjacent to the map block loaded, useless map blocks removed. For example, shown in Figure 6, set the initial display is 1, 2, 3, 4, 5, 6, 7, 8, 9 plots, when the characters move to the boundary of 6 plots, the system determines the parcel moves to the right, Plots 1, 4, 7 are removed, 3', 6', 9'will be loaded sequentially.

The museum scene is relatively large, so in the left side of the form to the thumbnail of the entire scene, and the user clicks the thumbnail of the venue, the display window can directly jump to the corresponding map area on the right side. When the user clicks the left side of the map area, this program could get the index number of the target area of the map. Since each map area is unique and the index numbers of the eight adjacent map areas around the map area are recorded in the XML, this program can find the final target map area. The program searches from the current map area to the outer layer. When the target index number is found, the search path is gradually traced back, and the path is sequentially stored in the sequence table, thereby realizing the jump operation from one map area to another map area.

\subsection{Full range of exhibits}

In this paper, a bright spot is the use of two-dimensional resources to achieve threedimensional effect. This method not only saves memory resources, but also achieves visual three-dimensional effect. In order to make it more clearly to the museum's exhibits, a small button is set on the exhibit's object in the 2.5-dimensional scene. The user clicks the button, which can view the exhibits in all directions model. In this design, the three-dimensional model 
of the exhibits is realized by the Draw Texture style of GUI. Firstly, the 3D model of the exhibits is built, and then the model of the 2.5 dimension exhibits at different angles is rendered. Adjust the coordinate axis of the model to the bottom center position, create a camera at (-50, $-50,50)$, resize it, and then render. In order to achieve the three-dimensional effect of cultural relics, so in the 3dsMax make the animation that artifacts rotate 360-degree; the user can observe the heritage by frame-by-frame rendering method. In order to achieve a good display of the three-dimensional effect of cultural relics without wasting memory resources, the purpose of this paper through experimental comparison 36 renderings. Tiger model renderings are shown in Figure 6. Because the model contains multiple items in the program, it needs to locate the 2.5-dimensional model corresponding to the model. First, get the index number of the browse model, and then get the corresponding path of the model, use the cycle to load 2.5 dimensional exhibits model image, each cycle, write the path of the 2.5 dimensional exhibits model, create WWW loaded object, using www.isDown () method to determine whether to complete the loading. Parameter list of exhibits three-dimensional observation parameters is shown in [Table 1].
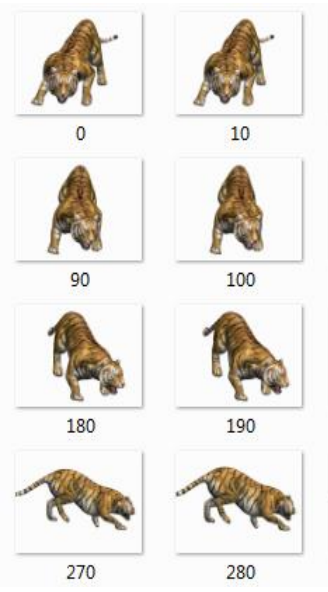

280
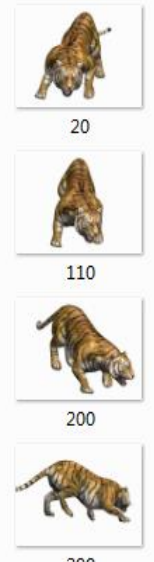

290
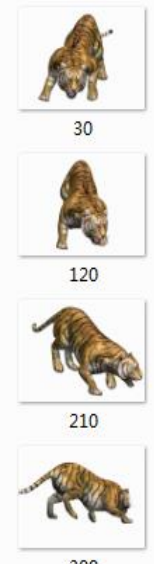

300
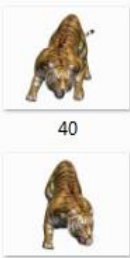

130
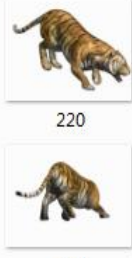

310

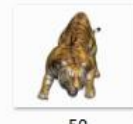

50

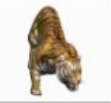

140

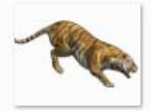

230

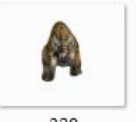

320
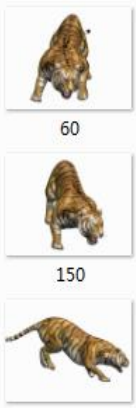

240

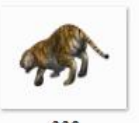

330
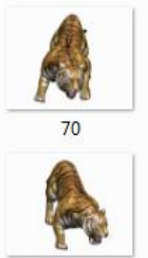

160

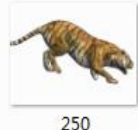

250
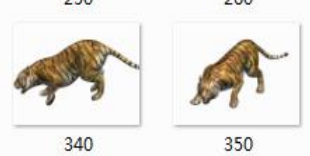

Figure 6. Tiger model renderings

Table 1. Parameter list of exhibits three-dimensional observation parameters

\begin{tabular}{|c|c|}
\hline Parameter & Description \\
\hline PicWidth & Width of exhibit model \\
\hline PicHeight & The height of the exhibit model \\
\hline Count & The total number of models \\
\hline Arr[] & An array of 2.5 dimensional models used to store loads \\
\hline CountI & The ith 2.5-dimensional exhibits model \\
\hline PathS & 2.5 dimensional model storage path \\
\hline Picurl & Loads the model's path name \\
\hline
\end{tabular}

In order to enable users to observe the details of the characteristics of the exhibits, the system contains the function of expansion and contraction. When the mouse wheel scrolls, the model image is zoomed to focus on the point where the mouse is clicked. During the display, a part of the image is sometimes displayed, so a window-to-view transition is required. Through the two-dimensional cutting process, the graphic data in the window which exists in the computer is mapped into the graphic data in the viewport area of the display through the window-view transformation, and the graphics outside the window are removed and not outputted. Move the 
center of the clipping window to the position of the mouse click, calculate the offset of the mouse relative to the view element by the internal variables client $X$ and client $Y$, and multiply the offset by the scaling factor to get the offset of the clipping window. According to the center coordinates of the cut window picture, the exhibit image is drawn by calling the DisplayCurImage () function. When the user to observe the details of the image at a certain angle, in order to make the user will not feel a longer Caton phenomenon, the system first zoom in thumbnails, and then load the high-definition image to replace the thumbnails to achieve the natural transition effect. The variables defined in DisplayCurImage () are shown in [Table 2].

Table.2 Function Variables

\begin{tabular}{|c|c|}
\hline Parameter & Description \\
\hline CurIndex & Current index point \\
\hline Centerx & the horizontal length of the midpoint of the image in the clipping window \\
\hline Centery & the vertical length of the midpoint of the image in the clipping window \\
\hline scale & The magnification of the image, the default is \\
\hline ClippingX & Abscissa of the vertices of the clipping window \\
\hline ClippingY & Vertical ordinate of the vertices of the clipping window \\
\hline CWidth & Width of the clipping window \\
\hline CHeight & Height of the clipping window \\
\hline ClippingCx & Abscissa of the midpoint of the clipping window \\
\hline ClippingCy & Vertical ordinate of the midpoint of the clipping window \\
\hline Vwidth & Width of viewing area \\
\hline Vheight & Height of viewing area \\
\hline
\end{tabular}

The function first determines whether the clipping window is out of bounds (cross-border problem as shown in [Figure 7]). The values of Centerx and Centery are determined by comparing the size of Centerx and ClippingCx and the size of Centery and ClippingCy. The processing method of the cross-border situation when the clipping window is in a different position is described in Fig.8. The relevant properties of the clipping window are determined by ClippingX, ClippingY, VWidth, VHeight four variables, where ClippingX, ClippingY determines its vertex position, CWidth and CHeight determine the width and height of the clipping window.

Abscissa of the vertices of the clipping window

$$
\text { Clipping } X=\text { center } x-\text { Clipping } C x
$$

Vertical ordinate of the vertices of the clipping window

$$
\text { Clipping } Y=\text { centery }- \text { ClippingCy }
$$

ClippingCx and ClippingCy can be determined by Vwidth, Vheight. The size of the clipping window is determined by the viewport. When the mouse to expand or shrink operation, it will directly affect the size of the crop window. The center point of the clipping window is converted to the center point of the viewport by the window-view transform, so the center point of the clipping window (ClippingCx, ClippingCy) is:

$$
\begin{aligned}
& \text { ClippingC } x=\text { Vwidth } \times \text { scale } / 2 \\
& \text { ClippingCy }=\text { Vheight } \times \text { scale } / 2
\end{aligned}
$$




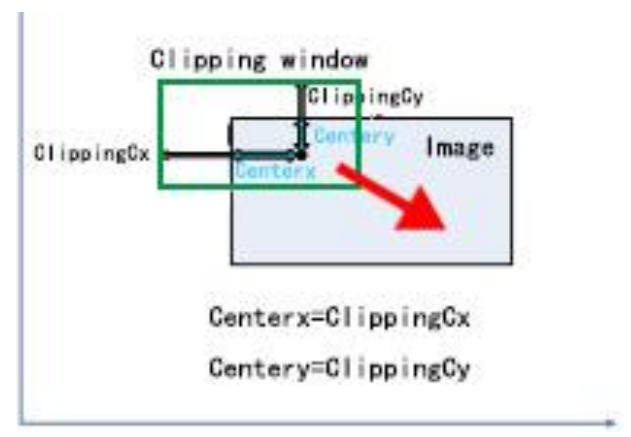

(a)

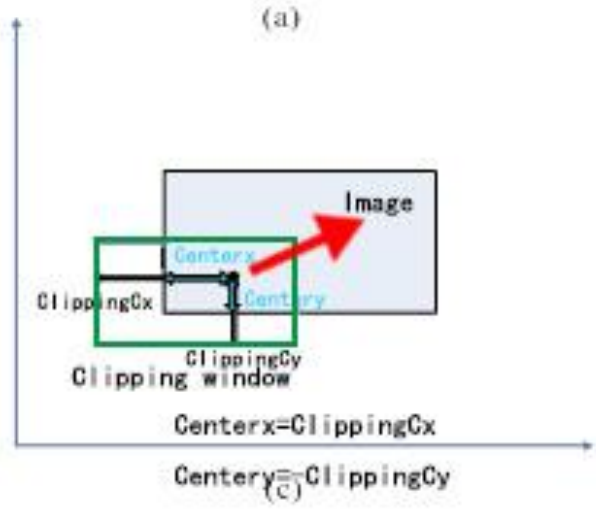

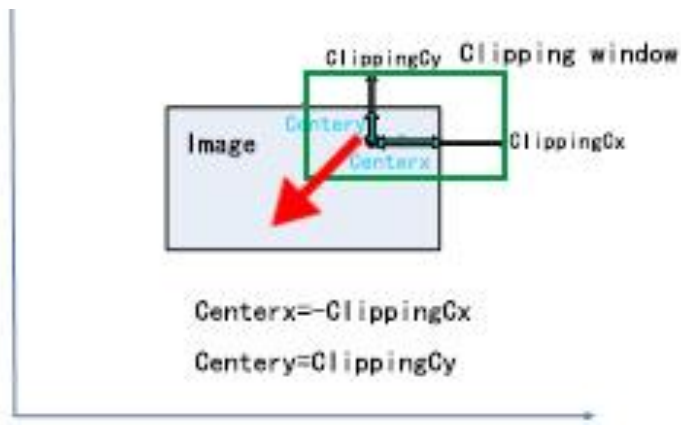

(b)

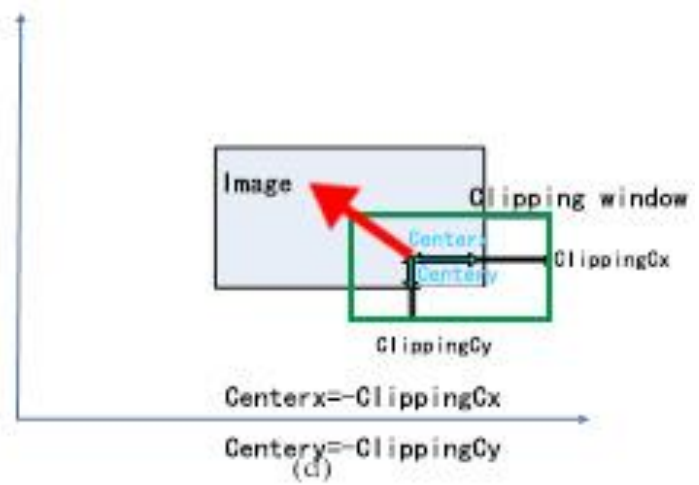

Figure 7. Mapping relationship between the clipping window and the graphics in the viewport

\subsection{Character tracking algorithm}

In this paper, the system designed by a virtual character role; the characters could walk in accordance with the user's instructions in map area. For the needs of environment, In order to meet the needs of the environment, the characters are designed by sit and walk. the characters are designed eight angles of action, which is $0^{\circ}, 45^{\circ}, 90^{\circ}, 135^{\circ}, 180^{\circ}, 225^{\circ}, 270^{\circ}, 315^{\circ}$, as shown in [Figure 8].

When the user clicks on the next target point, the virtual character according to the target point information to determine whether to change the direction of movement, whether to change the station, sit, go. When the coordinates of the target point and the coordinate of the current position, or reach the target point (except for the special region), the character is the status of the station; When the target position is not consistent with the current position, go; in the rest area, the program set up the target area for the sitting state. Whether or not the direction of motion is changed depends on the angle between the target point and the current position point in the horizontal direction. When the virtual character encounters obstacles, it changes the character's effect graph until it reaches the target point.

For example, as shown in [Figure 9], at present, the virtual character runs in the OA direction and the target point is A1.the current character running along the OA direction, the target point A1, If $\angle \mathrm{O} 1 \mathrm{OA}$ and the 8 aspects which are most close to the group call the corresponding image, if the closest angle O1OA, without reloading. 


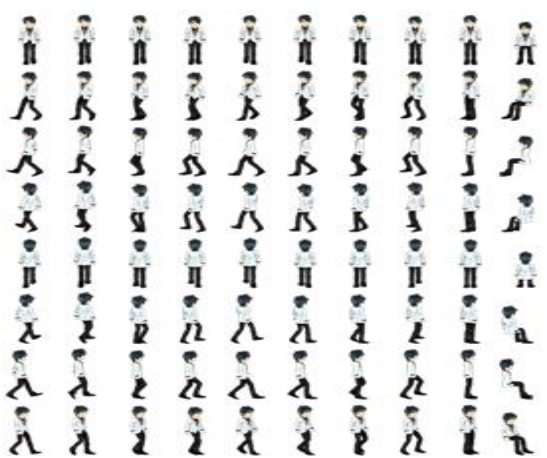

Figure 8. Character action

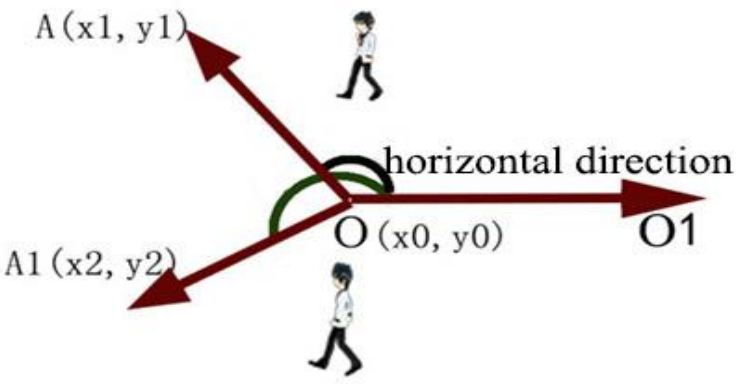

Figure 9. Schematic diagram

\subsection{Collision detection and depth detection of characters and stereoscopic objects}

\subsubsection{Collision detection}

When two objects with the Rigidbody2D attribute touch each other in the scene, they will have collision physical effects without penetrating each other. For example, character does not pass through booths and exhibits, people do not sink when walking, and so on. In this paper, collisions are implemented through collision effects in the physical engine of Unity3d. After the collision detection research, characters and Stereoscopic objects not only to achieve the effect of the collision, as well as the mutual occlusion effect. Therefore, when the characters collide with stereoscopic objects, the collision detection method is the characters and stereoscopic objects have Rigidbody 2D components and colliders. Character model has Polygon Collider 2D attributes and Rigidbody 2D attributes. The stereoscopic object is divided into two parts: the upper part of the stereoscopic object realizes mutual occlusion, only adds the Polygon Collider 2D; the lower part realizes colliding with each other and adds the Polygon Collider 2D and the Rigidbody 2D attribute. When the model is loaded into the scene, the Unity3d software will automatically identify the Polygon attribute of the imported model to achieve the impact of the collision. It is worth noting that the lower part of stereoscopic object cannot be triggered through, just a collision, and the upper part is the need for depth detection can be triggered through. The schematic of the collision is shown in [Figure 10].

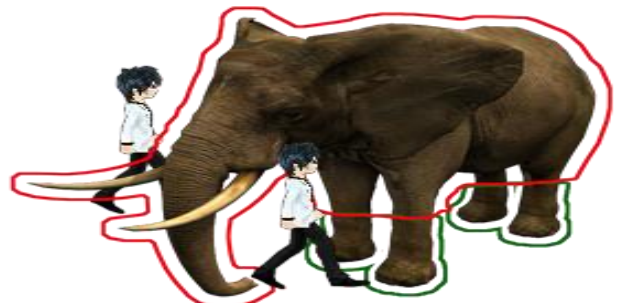

Character model(Polygon Collider 2D and Rigidbody 2D)

The upper part of sereoscopic object (Polygon Collider 2D)

The lower part of sereoscopic object (Polygon Collider 2D and Rigidbody 2D)

Figure 10. The schematic of the collision

\subsubsection{Depth detection}

Depth detection is used in Unity3d image layering technology, the same layer classification will not affect the Z-axis transformation, blocking effect by adjusting the depth of the order to achieve. The visual scene map is placed on the bottom layer and is placed on a single layer; the 
characters and the stereo objects are placed in the same classification layer. Layer sorting is different, so it can do collision detection between each other. When the characters collide with the three-dimensional objects, the system judges the layers of the characters and the stereo objects, and loads the stereo model to achieve the effect of collision blocking. The specific stratification is shown in [Table 3].

Table.3 Model of layered table

\begin{tabular}{|c|c|c|}
\hline Model & Sorting Layer & order in layer \\
\hline 2.5-dimensional scene map & Default layer & 0 \\
\hline Character model & Stereoscopic Objects layer & 0 \\
\hline Booth 1 Stereo Object Model & Stereoscopic Objects layer & 2 \\
\hline Booth 2 Stereo Object Model & Stereoscopic Objects layer & 4 \\
\hline Exhibit 1 Stereo Object Model & Stereoscopic Objects layer & 6 \\
\hline Exhibit 2 Stereo Object Model & Stereoscopic Objects layer & 6 \\
\hline$\ldots$ & Stereoscopic Objects layer & $\ldots$ \\
\hline
\end{tabular}

\section{Conclusion}

Visualization of museums is a window of the Internet. It is aiming at the problem of the low dimensional space and slow transmission in the visual management system of the museum. In this paper, a visual management system is based on 2.5 dimensions proposed. For speed up the loading speed of the network, this research set up pre-dynamic loading, and divided each map area into small plots: $256^{*} 256$. As [Figure 11], [Figure 12] is showed that the 2.5 dimensional visualization management systems is based on the Daqing City Museum as a prototype, which loads fast, three-dimensional sense is better. For some of the details of the management system, it is still need to be further studied.

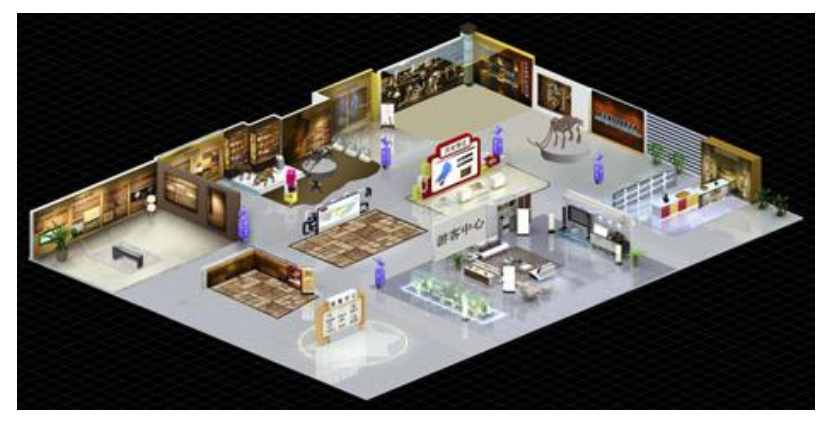

Figure 11. The 2.5 dimensional scene of an exhibition hall

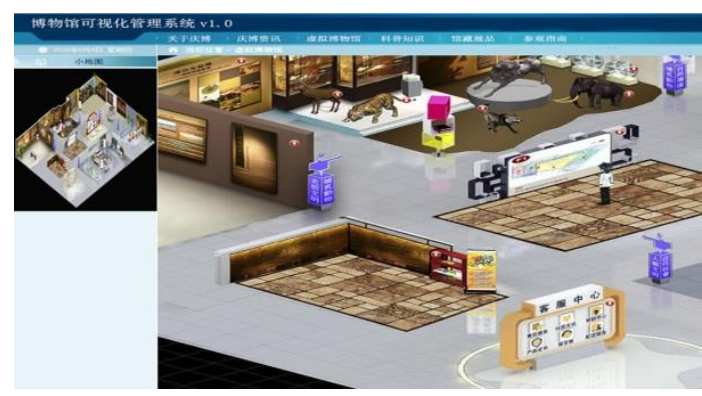

Figure 12. Visualization management 


\section{Acknowledgements}

The work is partially supported by the Daqing Municipal Science and Technology Project (szdfy-2015-37) and CAS Key Laboratory of Renewable Energy of China under Grant No.Y907k81001.

\section{References}

[1] Y.N. Lien, H.C. Jang, T.C. Tsai, P.J. Kuo, and C.L. Hu, "Mobilizing digital museum with chinese digital archive," The 8th IEEE International Conference on Ubi-Media Computing (IEEE UMedia'15), IEEE, (2015) DOI: 10.1109/UMEDIA.2015.7297443

[2] M.Q. Zhou, G. H. Geng, and Z.K. Wu. "Digital preservation technology for cultural heritage," Higher Education Press, BEI JING (2012)

[3] W. Ting, "Application of 3D digital modeling technology to cultural relics in the Museum of the Terra-Cotta Warriors and Horses of Qin Shihuang - Case study on terra-cotta warriors in Pit No.1," Sciences of Conservation \& Archaeology, no.4, pp.105-110, (2012) DOI: 10.16334/j.cnki.cn31-1652/k.2012.04.009

[4] U. Iqbal, P. Molchanov, T. Breuel, J. Gall, and J.Kautz, "Hand pose estimation via latent 2.5D Heatmap Regression," 15th European Conference, Munich, Germany, September 8-14, 2018, Proceedings, Part XI. Computer Vision - ECCV 2018, Springer, Cham, (2018)

[5] Liu H, "Design and implementation of intelligent community management system based on the 2.5 dimensions," Computer Technology and Development, vol.27, no.07, pp.156-169, (2017) DOI: 10.3969/j.issn.1673629X.2017.07.035

[6] G. Y. Liu, Y. Qi, Z. W. Lin, and G. C. Ding, "Transactions of the Chinese society of agricultural engineering," Transactions of the Chinese Society of Agricultural Engineering, vol.25, no.08, pp.164-168, (2009) DOI: 10.3969/j.issn.1002-6819.2009.08.030

[7] LIU G, GUO X, XI G, and LIU J, "Multi angle acquisition and 3D observation of insect specimen image," Computer Science, vol.43, no.6A, pp.239-241, (2016)

[8] Y. Y. Li, G. Y. Li, and T. C. Yu, "Agricultural machinery visual management platform construction base on the 2.5 dimensions space," Journal of Chinese Agricultural Mechanization, vol.37, no.02, pp.200-204, (2016) DOI: 10.13733/j.jcam.issn.2095-5553.2016.02.045

[9] W. XU and L. Wang, "Discussion on the method of 2.5-Dimensional electronic map rendering," Geomatics \& Spatial Information Technology, vol.40, no.10, pp.202-204, (2017)

[10] L. ZHANG and Z. SI, "The design and implementation of 2.5 dimensional campus map based on web," Computer Knowledge and Technology, vol.12, no.02, pp.80-81, (2016) DOI:10.14004/j.cnki.ckt.2016.0147

[11] X. Qu, J. Zhou, B. fu, and J. Ning, "In-time map generation method for 2.5d game," Journal of System Simulation, vol.18, no.1, pp.414-416, (2006) DOI: 10.3969/j.issn.1004-731X.2006.z1.128

[12] X.Yu, X. Zhang, L. Liu, J. N. Hwang, and W.Wan, "Dynamic scheduling and real-time rendering for largescale 3d scenes," Journal of Signal Processing Systems, vol.75, no.1, pp.15-21, (2014) DOI: 10.1007/s11265013-0860-1

\section{Authors}

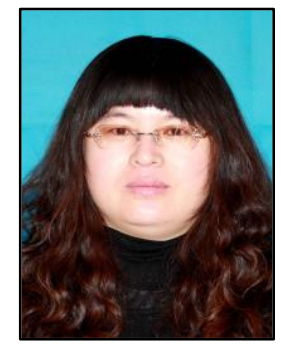

\section{Na Wang}

$\mathrm{Na}$ Wang is currently a lecturer at Heilongjiang Bayi Agricultural University. He received master's degree in Shenyang University of Technology. Her research work is the application of information technology in agriculture. 